Cahiers d'études africaines

198-199-200|2010

50 ans

\title{
Singeries au Congo
}

Monkey business in Congo

Nicolas Martin-Granel

\section{OpenEdition}

Journals

Édition électronique

URL : https://journals.openedition.org/etudesafricaines/16514

DOI : 10.4000/etudesafricaines. 16514

ISSN : 1777-5353

Éditeur

Éditions de l'EHESS

\section{Édition imprimée}

Date de publication : 20 novembre 2010

Pagination : 1113-1145

ISBN : 978-2-7132-2252-8

ISSN : 0008-0055

\section{Référence électronique}

Nicolas Martin-Granel, « Singeries au Congo », Cahiers d'études africaines [En ligne], 198-199-200 |

2010, mis en ligne le 02 janvier 2013, consulté le 23 avril 2022. URL : http://journals.openedition.org/ etudesafricaines/16514; DOI : https://doi.org/10.4000/etudesafricaines.16514

Ce document a été généré automatiquement le 23 avril 2022

(C) Cahiers d'Études africaines 


\title{
Singeries au Congo
}

\author{
Monkey business in Congo
}

Nicolas Martin-Granel

\section{NOTE DE L'AUTEUR}

Une première ébauche de cet article est parue en anglais, voir MARTIN-GRANEL (2001).

1 Pour situer et illustrer d'entrée de jeu ce que j'entends par «singeries ", qu'on me permette de commencer par deux anecdotes entendues au Congo. La première relève d'une expérience personnelle : à mon arrivée à Brazzaville en 1990 après des années passées en Afrique de l'Ouest, j'ai été frappé d'entendre, d'une part, des résidents français dire banalement et d'une manière fort candide : "Dès que nous serons rentrés chez nous, ils remonteront aux arbres... », et, d'autre part, de voir, dans certaines boutiques de commerçants, la même affichette portant l'inscription: "Si moi faire crédit / Toi pas payer moi fâché / Si moi pas faire crédit / Toi payer moi content / Moi pas faire crédit. » On tient là, sur le mode grotesque, les deux branches principales du syndrome de la singerie, d'un côté l'ancestralité simiesque qui renvoie via la vulgate darwinienne au mythe des origines et, de l'autre, le petit-nègre considéré comme le chaînon manquant entre les « patois » africains et la langue française.

2 La seconde anecdote montre comment l'imagerie raciste a pu être "assimilée » par ceux-là mêmes qui en étaient les victimes. Il s'agit de l'usage scolaire du " symbole ». L'écrivain Sony Labou Tansi a plusieurs fois raconté (Martin-Granel 1995 : 764) que les maîtres de l'école primaire coloniale suspendaient une boîte remplie d'excréments au cou des mauvais élèves qui commettaient des fautes de français ou celle de refuser de parler cette langue considérée alors comme signe distinctif des «évolués ». Pourtant "l'évolution» vers l'indépendance n'a pas fait disparaître ce bonnet d'âne particulièrement infamant. En effet, un jeune collègue universitaire me disait que dans son école on accrochait au cou des cancres un crâne de singe - un "symbole ", on le voit, on ne peut plus symbolique... 
3 Retenons l'emploi curieux du terme "symbole", en songeant à ce qu'il signifiait à l'origine : chez les anciens Grecs le mot symbolon désignait ce bout de bois que les deux hôtes - celui qui offre l'hospitalité comme celui qui la reçoit - cassaient en deux parties au moment de se séparer et dont chacun gardait une moitié de façon à ce que, le jour où ils se rencontreraient à nouveau, ils puissent se reconnaître en rajustant les deux morceaux. Le crâne de singe nous servira donc de signe de reconnaissance (au second degré) de ce qui s'est noué en Afrique centrale entre l'imaginaire du Blanc esclavagiste et colon, et celui du Noir autochtone. Celui-ci a reçu celui-là, souvent à son corps défendant, et a gardé en dépôt le symbole de l'hospitalité inégale et raciste. La blessure de ce rendez-vous manqué ne s'est pas cicatrisée, l'injure s'est conservée dans le symbole simiesque, un «symbole» non plus, comme chez les Grecs, de reconnaissance et d'amitié mais de méconnaissance et de xénophobie.

On ne citera ici que deux exemples de la persistance de ce syndrome simiesque ${ }^{2}$. Voici d'abord ce que disait Sony Labou Tansi en $1987^{3}$ :

"On leur a fait croire en face, à Kinshasa, que pour être considéré comme un homme, il fallait avoir un certificat d'évolué ; c'est-à-dire, à ce moment-là, tu étais comme un Blanc. Tu étais évolué et ça veut dire que tu es sorti des singes [...]. À la campagne, ceux qui travaillaient la terre étaient des macaques, des singes. C'est ça qui a existé. Alors cet homme qui est venu en ville continue à se prendre pour l'évolué et il continue à prendre les gens qui sont restés à la campagne pour des singes. ")

5 Ces propos montrent bien comment le racisme colonial a pu être assimilé et imité par les "évolués » qui ont ainsi reproduit les préjugés de leurs maîtres. Les catégories sociales non urbanisées sont ordinairement désignées par le terme péjoratif de "villageois ", et comme elles ne parlent pas français, elles s'expriment en "patois". Mais l'écrivain ne mâche pas ses mots : il n'a pas « votre honte d'appeler les choses par leur nom ». Derrière "villageois » et " patois », il retrouve l'injure primitive qu'il met ainsi à nu : mossendzi (mon singe) $)^{5}$. La reconduction de la métaphore insultante - et qui s'applique à présent à des frères et même à soi-même - mène à une autodépréciation qu'on pourrait appeler autoracisme ${ }^{6}$.

Notre second exemple concerne la façon dont a été ressentie à Brazzaville l'affaire de l'expulsion des «sans-papiers» de l'église Saint-Bernard à Paris. Le journaliste d'un hebdomadaire satirique écrit :

«Le Seigneur a dû fermer l'œil : après tout ce n'était que des africains noirs avec beaucoup de Musulmans parmi eux. Tant pis! La France opulente n'est pas là à héberger les misères des autres. Et oust ! coup de pied au cul! Rentrez chez vous, bande de macaques! $»^{7}$.

7 Ce qui grimace encore dans cette ironie grinçante, c'est le singe auquel renvoient une hospitalité manquée et une humanité déçue. La figure du singe est le symbole d'un lieu où les cultures s'ajustent sans se rencontrer. Il y a comme un "différend", au sens où l'entend le philosophe Jean-François Lyotard, c'est-à-dire un «malentendu à certains égards insurmontable entre des réclamations rédigées dans des idiomes hétérogènes ». D'autant plus «insurmontable », en l'occurrence, que le prétendu «singe » est réduit soit au cri sauvage de l'animal, soit au "patois» barbare, soit encore, comme ici, à singer ironiquement l'idiome de l'Autre en mimant son insulte favorite: «bande de macaques!» 


\section{Le Congo, berceau de l'homme-singe}

8 Un peu partout, au Nord comme au Sud, on dit que l'Afrique est le berceau de l'humanité. L'image est devenue un cliché qui se colporte et se propage avec d'autant plus d'assurance qu'elle est censée avoir la caution de la Science. Depuis la découverte de la fameuse Lucy, les archéologues, les anthropologues, les paléontologues ne cessent de se pencher sur ce berceau et de le remplir de leurs trouvailles et de leurs controverses. Par-dessus leurs épaules, les médias lisent le texte de l'origine de l'homme et vulgarisent la bonne nouvelle du passage de l'animalité à l'humanité, et notamment du singe à l'homme.

Cependant, à l'occasion de ce passage, des fantasmes resurgissent et la vulgarisation scientifique produit en écho une vulgate mythologique. C'est par exemple le fameux "chaînon manquant» qui accrédite la représentation mythique d'une évolution naturelle qui se serait faite sans mutation, comme si on était passé de l'animal à l'homme sans solution de continuité. À la limite l'espèce humaine finit par se confondre avec les espèces animales, par être ramenée à l'animalité primitive. Cette confusion se remarque explicitement dans les termes désignant les spécialistes scientifiques: le zoologue ou le primatologue ne se distingue guère de l'anthropologue ou de l'ethnologue.

10 Or, ce genre d'amalgame est favorisé par l'imaginaire de la forêt primaire considérée comme la niche écologique primitive. À l'ombre de la forêt profonde et sauvage, comment discernerait-on les primates des hommes? C'est la loi de la jungle, où tout le monde est tour à tour chasseur et gibier.

11 Toute une littérature venue du Nord se penche sur le berceau africain de l'humanité, avec des préoccupations écologiques ou des préjugés racistes. Mais il arrive que ces deux cadres idéologiques se superposent, comme dans un petit texte qui n'a certes rien de littéraire mais qui apparaît comme un condensé symptomatique des stéréotypes dans lesquels puisera le corpus littéraire que nous examinerons par la suite. Il s'agit d'une publicité pour les véhicules Toyota que l'on pouvait lire il y a quelques années dans des hebdomadaires français. Ce qu'elle met d'abord sous les yeux, ce n'est pas l'image attendue de la technologie nippone, mais un troupeau de zèbres figés par un cliché du style safari. En dessous, en guise de «légende » (au double sens du terme), on lit une phrase qui pastiche le genre oral du conte énigmatique à tiroirs: «Voici les zèbres qui ont vu le lion qui a vu l'homme qui avait une Toyota. » Suit enfin l'histoire de cet homme contée comme la légende du martyr de l'écologie :

«Mais l'homme à la Toyota, qui est garde-chasse au Kenya dans la réserve de Masaï Mara, sait que si ça continue, il n'y aura bientôt plus rien à voir dans les belles réserves d'Afrique. Disparaissent les éléphants, les rhinocéros, les gorilles [...]. Et bientôt, si l'on n'y prend garde, ce sont des dizaines d'espèces qui n'existeront plus que sur les planches des encyclopédies avec la mention "disparu". L'homme à la Toyota traque les braconniers qui font commerce de l'ivoire et autres trophées; au risque de sa vie, car on ne compte plus les ethnologues ou les gardes-chasses assassinés par des trafiquants. L'homme à la Toyota connait tout de la faune qu'il est chargé d'approcher pour mieux la protéger, de la réserve et des Masaïs qui y vivent. "

12 Cette défense et illustration du patrimoine naturel intriguent déjà dans une publicité destinée à promouvoir des engins polluants et, qui plus est, produits par un pays réputé gros consommateur d'ivoire... Mais il y a plus inquiétant: l'emploi du terme 
« ethnologues » à propos de la faune. Qu'il s'agisse d'un lapsus calami ou d'une erreur de traduction, il révèle de bien étranges confusions et soulève certaines questions cruciales, car que viennent donc faire ces ethnologues parmi les zèbres? Étudier «l'ethnie » des gorilles ? À moins que ce ne soient les gardes-chasses qui protègent les Masaïs, une espèce en voie de disparition comme les autres animaux de la réserve ? C'est d'ailleurs sans doute ce terme de "réserve» qui favorise le brouillage de la frontière entre animalité et humanité, ainsi que celle, corrélative, qui sépare sciences de la nature et sciences de l'homme.

"L'homme à la Toyota ", en effet, semble bon à tout faire et à tout savoir sur les bêtes et sur les hommes, à l'aise aussi bien sur le terrain de l'ethnologue que sur celui de l'éthologue.

Cette page publicitaire peut donc être prise comme une fable exemplaire. On y voit en quoi l'arbre idéo-écologique cache la forêt aux arbres d'essences diverses, la forêt aux animaux d'essences diverses et même la forêt habitée par des hommes aux cultures tout aussi diverses. C'est la zoophilie, la maladie infantile de l'écologisme, qui confond toutes ces forêts pour en faire un lieu unique et mythique, infernal ou paradisiaque, à la fois berceau et tombeau de l'animal, tantôt sous-homme tantôt surhomme. Or, cette forêt est aussi bien celle du Congo que du Kenya - les Masaïs étant alors remplacés par les Pygmées, comme on le verra par la suite.

\section{Cette forêt qui cache le singe}

Souvenons-nous d'abord que le mot "forêt " provient du latin médiéval silva forestis (forêt qui dépend du tribunal royal). Il désigne à l'origine « une réserve de chasse », ou encore « une vaste solitude où se reproduisent les bêtes sauvages "; les hommes ne s'y aventurent que pour « s'y marginaliser, s'y conduire en l'homme de la nature, fuyant le monde de la culture dans tous les sens du mot » (Le Goff 1980).

Cependant «le grand thème médiéval de l'homme sauvage " resurgit avec force à l'époque contemporaine, et cette résurgence a lieu en Afrique, le dernier lieu primitif qui reste à l'Occident. Où sont en effet les forêts d'antan sinon au cœur de l'Afrique ? Au Congo précisément, même si ce Congo ne correspond à aucune entité géographique précise et n'existe que dans «la topographie psychique » de l'explorateur. La forêt congolaise forme un ensemble flou, dont les contours sont comme noyés dans les couleurs du rêve et de l'aventure.

17 C'est d'abord une terra incognita, une sorte d'Amazonie africaine, représentée par une tache blanche sur les cartes de l'Afrique du xix ${ }^{e}$ siècle. Ou bien c'est le noir - la supposée couleur locale - qui s'impose tout naturellement en une sorte de synecdoque généralisante! C'est du moins ce qui est suggéré dans l'introduction du roman de Michael Crichton (1990) intitulé Congo : «En fait, on appelle l'Afrique le continent noir pour une seule et unique raison: les vastes forêts équatoriales humides de sa partie centrale.»Et plus loin, l'auteur nous explique que ce que les anciens Égyptiens appelaient le Pays des Arbres était « un endroit mystérieux, couvert de forêts si denses qu'en plein jour il faisait aussi noir qu'en pleine nuit ${ }^{8}$. D'étranges créatures habitaient cette obscurité perpétuelle, y compris de petits hommes pourvus de queue et d'animaux mi-noirs, mi-blancs. » On pressent alors toute la matière romanesque que recouvre ce nom mythique de Congo, au-delà de l'apparence sobrement géographique du titre, comme le souligne d'ailleurs l'auteur: «En fait, en 1979, le mot Congo 
désignait techniquement le système hydrographique du fleuve Zaïre, encore qu'on l'appelât toujours Congo dans les milieux des géologues, par familiarité et pour le côté romanesque de l'appellation. » On verra plus loin en quoi le Congo fait en effet tout un roman, voire tout un film ${ }^{9}$.

Pourtant il arrive qu'un récit de voyage parle du Congo réel avec des couleurs plus réalistes. Le pays n'est plus ni blanc ni noir; il prend la couleur naturelle, écologie oblige. L'auteur de ce récit, Patrice Franceschi (1991) ${ }^{10}$, écrit :

«Passionné depuis longtemps par les "taches vertes" en général et par celles de l'Afrique Équatoriale en particulier, je décidai vers la fin de l'année 1974 de réunir trois de mes anciens compagnons d'Afrique afin de monter une expédition dans une région la plus mal connue du Nord Congo. »

Le livre porte un titre accrocheur: Au Congo jusqu'au cou. Le sous-titre ne l'est pas moins, dans sa grandiloquence épico-journalistique : « Expédition Babinga-Pongo (juinoctobre 1975), ou l'aventure initiatique et extrême de quatre Français de 20 ans chez les Pygmées de la forêt équatoriale. » Pour être jeune, l'aventurier des temps modernes ne découvre rien de bien nouveau; il suit le chevalier du roman médiéval dont les traces mènent au cœur de la forêt repoussante et désirable, dans ces «lieux de l'extrême marge où l'homme peut s'aventurer et y rencontrer d'autres hommes - à la limite ces hommes sauvages qu'il prend d'abord pour des bêtes lui affirment qu'ils sont des hommes ». C'est sans doute pourquoi, comme Franceschi nous l'explique naïvement, "l'expédition fut bientôt baptisée "Expédition Babinga-Pongo" (Babinga = Pygmée, pongo $=$ lutter, survivre et c'est aussi le nom du gorille) ». Passons sur ce jeu de mots qui voudrait nous faire douter de l'humanité des Pygmées, assimilés, sous couvert de la loi de la jungle, à des pongidés. On reviendra sur ce « détail » qui est pourtant de taille, mais dans l'immédiat revenons à ces jeunes explorateurs perdus, comme il se doit, dans la forêt primaire - primitive pour eux - du Congo.

Celle-ci ne garde pas toujours la belle couleur verte qu'elle avait au départ, sur la carte. C'est une forêt caméléon qui change en fonction de l'humeur du spectateur. D'abord, «nous découvrons tout un univers qui s'étend en nuances infinies du répugnant au merveilleux ». La merveille l'emporte quand on peut la tenir à distance, en prendre des images qui sont autant de clichés : « Un spectacle fait d'arbres, de lianes, de racines, de fleurs. Une vaste fresque, immense et chatoyante d'un vert qui prend toutes les teintes que peut imaginer l'esprit. » Forêt aussi merveilleuse, ici, que le sourire des Pygmées "empreint de naïveté et d'innocence", et d'autant plus merveilleuse aux yeux du voyageur qui imagine «tous ceux qui auraient voulu partir et qui peut-être en cet instant se bousculent dans le métro, pauvres fourmis pâles et besogneuses ». Mais dès que les vraies fourmis de la forêt viennent jouer le rôle de trouble spectacle, la couleur sombre reprend le dessus : "La jungle n'est pas un enfer à cause des grands animaux. L'enfer, c'était les insectes, les milliers d'insectes, minuscules et voraces, qui s'insinuent partout et obligent à une lutte incessante, jour et nuit jusqu'à l'obsession. » Céline avait parlé de cette obsession des fourmis voraces, avec l'émotion littéraire qui la faisait partager. Mais ici, dans Au Congo jusqu'au cou, l'évocation de la jungle, infernale pour l'homme blanc, ne sort pas du bourbier des métaphores hyperboliques et stéréotypées: " immense caverne sombre", "grotte obscure aux mille ramifications trompeuses et traitresses ", " univers dantesque ", etc. Un bourbier dont on ne saurait d'ailleurs sortir, puisqu'on y entre par le merdier primordial impliqué dans le titre : ... jusqu'au cou - à la manière dont l'écolier africain entre en langue française par le symbole... ${ }^{11}$ 
21 Le récit de voyage n'échappe pas plus que la fiction à la prégnance du mythe fondateur du Congo profond. Ce mythe a une double généalogie, littéraire et coloniale, avec ces deux figures éponymes : Conrad et Stanley. L'écrivain remontant le fleuve à partir de la côte Atlantique, et, à l'opposé, le journaliste explorateur découvrant le Pool, devenu le Stanley Pool, en provenance de la Côte est. Le bassin du Congo se trouve en quelque sorte pris en tenailles des deux côtés, à l'est et à l'ouest, par ces deux ancêtres de l'exploration occidentale. Tout se passe comme si on ne pouvait que repasser sur leurs traces; les voies qu'ils ont frayées pour la première fois seront empruntées par les futurs voyageurs venus du Nord, qu'ils soient journalistes, écrivains, missionnaires religieux ou scientifiques, ou bien encore personnages de roman, et parfois un peu tout cela à la fois. Ainsi, Gide et Naipaul, dans leurs notes de voyage, se réclament de Conrad $^{12}$, tandis que Lennart Hagerfors (1990), un auteur suédois qui a passé son enfance au Congo, prend Stanley comme héros de son roman, Les Baleines du Lac Tanganyika.

22 Les deux voies de pénétration se croisent chez Graham Greene (1960) : un artiste connu remonte le fleuve pour enterrer sa célébrité " au cœur des ténèbres ", tandis qu'en sens inverse un journaliste sans scrupules vient l'inonder de la fausse lumière du cliché colonial : « La forêt éternelle somnole au long des rives inchangées depuis que Stanley et sa petite troupe [...]. » Le cliché journalistique fond toutes les forêts dans le singulier générique de LA forêt, forcément africaine, nécessairement congolaise.

Cette vision monochrome de la forêt nous permet de rapprocher les deux branches de cette Amazonie africaine qui sert d'arrière-plan à deux romans contemporains : Congo de Michael Crichton (1990) et Brazzaville Plage de William Boyd (1991). Le premier aborde la forêt par son versant oriental et nous en donne une version marquée par les premiers pas du conquistador blanc Stanley; la forêt du second est proche du littoral atlantique et baigne dans l'atmosphère conradienne du « retour aux premiers âges de l'humanité ». Cependant, leur titre même les situe dans le même pays imaginaire, un Congo sans frontières, utopique et atopique, où tout se confond, la nuit avec le jour, le Masaï avec le Pygmée, l'homme avec le singe et même le gorille avec le chimpanzé. Dès lors, le Congo prend une extension géo-littéraire élargie à tous les pays du Bassin ${ }^{13}$, creuset conceptuel et consensuel de la primitivité qui accueille pêle-mêle "gorilles, cannibales et Pygmées » avec leur cohorte de primatologues et d'ethnologues, «cœur d'Afrique $»^{14}$ embrassant tous les genres d'habitants et de visiteurs habités de toutes sortes de fantasmes, scientifiques ou touristiques.

\section{Le singe, face cachée de l'homme}

Comme la nature, étant la Nature même, la forêt a horreur du vide, et la forêt romanesque encore plus, qui a besoin de personnages à agiter devant son décor monotone. Les papillons de Gide sont bien trop légers, les Pygmées de Franceschi donnent trop « l'impression de bêtes de zoo ».

Le singe est évidemment le mieux placé pour imiter - on dit bien «singer " - ce que l'homme fait d'ordinaire dans les romans (tuer et se faire tuer, aimer et haïr...) ; il jouera donc le rôle de premier personnage de la forêt. Outre le fait qu'il représente l'habitant le plus vraisemblable de par son autochtonie, il présente des états de service anciens et convaincants dans le même rôle. Aussi, avant d'examiner pour quelles fonctions précises nos deux romans de référence, Congo et Brazzaville Plage, ont recruté 
respectivement des gorilles et des chimpanzés, il convient de rappeler qu'ils ne font que reprendre du service.

Le premier récit de voyage en Afrique à faire mention des hommes-singes remonte à l'Antiquité grecque. Il s'agit du célèbre Périple d'Hannon, dans lequel un commentateur moderne (Jacob 1991) voit «le paradigme du voyage d'exploration, rythmé par la temporalité linéaire des grandes navigations, régi par la subtile gradation qui conduit du dépaysement de l'exotisme au choc de l'altérité absolue ». Dans la logique de cette représentation imaginaire, le comble de l'étrangeté se trouve au bout du voyage :

« Durant trois jours, à partir de là, nous naviguâmes auprès de ruisseaux ardents. Nous arrivâmes au golfe nommé la Corne du Sud. Dans l'enfoncement se trouvait une île, semblable à la précédente, contenant un lac à l'intérieur duquel il y avait une autre île, pleine d'hommes sauvages. Beaucoup plus nombreuses étaient les femmes. Elles avaient le corps velu et les interprètes les appelaient Gorilles. » Trois brèves remarques sur ces « Gorillas ». - Le texte les considère non comme des animaux mais comme des êtres humains de sexe féminin, comme c'était le cas d'une autre peuplade mythique, les Amazones. - L'adjectif «velu» qui qualifie leur corps à la pilosité anormalement développée, s'applique aussi en grec à la végétation d'une forêt dense.

- Le nom «Gorilles" n'est attesté nulle part ailleurs que dans ce texte, et il est probable qu'il est dû à l'erreur du copiste qui aurait écrit "Gorillas» au lieu de "Gorgadas ", c'est-à-dire " Gorgones », autres créatures monstrueuses de la mythologie grecque: «Une très ancienne tradition, en effet, localise au sud-ouest de l'Afrique le séjour des Gorgones et le théâtre de l'affrontement entre Persée et Méduse. »

31 Mais les mythes ont la vie dure, ils constituent "le décor mythique » du roman moderne. Aussi n'est-il pas difficile de reconnaître la figure de Méduse, la Gorgone des Gorgones, dans l'histoire que Crichton nous conte dans Congo. Seuls les moyens et les armes ont changé : les Persées modernes sont des hommes de science, voire de sciencefiction, et c'est armés de la technologie la plus sophistiquée qu'ils se défendent contre de nouvelles Méduses, des créatures d'autant plus terrifiantes que, malgré la mémoire des ordinateurs, le savoir de la science est impuissant à les identifier, et, pour les désigner, en est réduit à revenir au nom antique pour forger l'adjectif " gorillesque » :

«Voilà que l'expédition prenait une nouvelle tournure, dans le sens d'un danger encore plus grand. Son premier mouvement de renoncement s'effaça au souvenir de l'écran vidéo et de la créature grise "gorillesque" dont il pensait qu'il s'agissait d'un animal nouveau, inconnu. Cette découverte-là valait le risque."

32 À vingt siècles de distance (le Périple d'Hannon date du $\mathrm{I}^{\mathrm{er}}$ siècle avant J.-C.), c'est toujours le même discours, marqué par la volonté d'explorer «les confins de l'humanité ». Avant d'en venir aux avatars les plus récents de ce discours, je voudrais juste mentionner deux étapes intermédiaires, deux variations sur le thème de l'hommesinge.

33 La première appartient à la littérature coloniale. Dans une nouvelle publiée à Bruxelles en 1900, Gim, le personnage-titre, est arrivé en Europe « vers la douzième année de sa vie, dépourvu d'état-civil mais porteur de son petit cœur rouge, son cœur d'animal noir ». La suite est à l'avenant, ouvertement raciste :

«Bibelot rare, cette fleur de chair exotique, une chose entre le singe et l'homme.

[...] petite bête amusante, venue d'un pays où cessait l'humanité générale, où la 
distinction entre l'homme et le singe était si peu sensible qu'il valait autant n'en point parler ».(cité dans Quaghebeur 1992) donc bien placée pour observer la métamorphose subie par Livingst
celui-ci est décrit lors de ses légendaires retrouvailles avec Stanley :

«Les jeunes gens soutenaient un vieillard qui ressemblait à un chimpanzé. Les jambes étaient maigres et arquées, les bras minces comme si quelqu'un les avait étirés. Les cheveux et la barbe étaient gris et hirsutes, le trou de la bouche s'ouvrait et se refermait sans bruit [...]. L'un des garçons arabes saisit le bras droit du vieux et lui fit tendre une main qui ressemblait à une griffe. Stanley la prit entre les deux siennes, puis il tomba à genoux, baisa la patte griffue et la serra en pleurant contre sa joue.

Stanley se releva lentement.

- Docteur Livinstone, je présume.

L'ancêtre eut un bref éclat de rire qui ressemblait à un aboiement, mais la panique le reprit aussitôt. Stanley passa le bras raide et simiesque du vieux autour de ses épaules et reconduisit Livingstone à sa hutte. »

Dans cette vision négative qui prend évidemment le contre-pied de l'anecdote historique, les hiérarchies s'inversent : le surhomme apparaît comme un sous-homme, le héros comme un singe. Même le grand Stanley n'échappe pas au devenir-animal qui est la loi de la jungle. Et quand son fusil rate la cible et que le gibier lui échappe, Shaw note: «Je crois pour la première fois qu'il avait honte de ne pas être un animal »tandis que les singes, eux, sont dans le devenir homme :

"Les singes ont quelque chose de Stanley. Il faut toujours qu'ils exagèrent. Dès qu'ils nous voient, ils se mettent à pousser des hurlements à propos du déclin du monde et de la chute des corps célestes. »

C'est précisément à cette remontée paradoxale du singe vers l'homme que s'intéressent un certain nombre de romans et de récits parus dans ces années-là. On mentionne d'abord pour mémoire deux romans des années 1980 qui, certes, ne situent pas des 
singes dans le bassin congolais mais lancent le thème qui sera exploité plus à fond dans le « Congo profond ${ }^{17}$ par nos deux romans « congolais ».

Le premier est White Spirit de Paule Constant (1989). L'action se passe quelque part en Afrique, dans une bananeraie; elle met en scène "trois innocents", dont Alexis le chimpanzé. De lui, le prière d'insérer dit qu'il « ne sait pas qu'il est un singe ».

La même année que la traduction française des Baleines du Lac Tanganyika, paraît l'admirable roman de Michel Rio (1985), Les Jungles pensives. Ce qu'en dit la quatrième de couverture est suffisamment explicite pour notre propos:

« Afrique, 1913. Un jeune biologiste (le narrateur) débarque en Côte d'Ivoire pour diriger une expédition scientifique dont la mission est d'étudier le comportement des chimpanzés dans leur milieu [...].

Europe, 1914. L'élite de l'espèce supérieure s'est déclarée à elle-même une guerre d'extermination. Le narrateur, sortant de la sauvagerie intelligible de l'ordre naturel, entre dans la sauvagerie sans mesure de l'ordre social. D'observateur des choses il devient jouet de l'Histoire. Le meurtre, planifié par l'intelligence, se fait illimité. De quoi rendre les jungles pensives. »

Ainsi est posé, entre le singe et l'homme, supposés, respectivement, "sauvage " et "civilisé ", le parallèle à partir duquel une réflexion éthique, philosophique et scientifique insérée dans la trame romanesque va opérer des renversements de perspective dont le résultat le plus évident est le brouillage de la frontière entre les espèces humaine et simiesque.

On peut passer rapidement sur Au Congo jusqu'au cou, où la réflexion sur le thème de l'homme singe ne dépasse pas les quelques naïvetés stéréotypées qu'il est d'usage de rencontrer dans les récits de voyage ${ }^{18}$. Ainsi, lorsque les jeunes explorateurs se trouvent face à un chimpanzé dont ils viennent de blesser la femelle, leur réaction consiste à anthropomorphiser l'animal : "Nous gardons la cruelle impression d'avoir un homme en face de nous, à la fois furieux et désespéré. » Ailleurs, le lecteur a l'impression qu'ils assimilent l'acte de manger du singe - courant chez les peuples de la forêt - à du cannibalisme, et que celui-ci équivaut à une sorte de péché originel dont le châtiment immanent serait un (re)devenir animal inéluctable :

«Une sorte de folie nous prend [...]. Nous nous sentons devenir des fauves impitoyables [...]. Pour boire, il nous faut nous traîner jusqu'à la rivière comme des animaux. »

\section{Deux portraits de singes en personnages de roman}

Autrement plus consistantes sont les histoires de singe que nous racontent Congo et Brazzaville Plage. Soit deux romans anglophones ${ }^{19}$ des années 1980. Le premier, qui ouvre la décennie, est de 1980, le second de 1991. Outre ces ressemblances externes, et en plus de leur commune référence congolaise affichée dès le titre, il est troublant de constater un certain nombre d'analogies.

En premier lieu, ils inscrivent d'emblée, dans l'épigraphe, le souci humaniste du rapport du Moi et de l'Autre. Michael Crichton met en exergue une réflexion désabusée de Stanley, qui situe l'homme d'action dans la tradition des moralistes:

«Plus je tire d'expérience et de discernement de la nature humaine, plus j'acquiers

la conviction que la partie la plus essentielle de l'homme est purement animale. »

Quant à Brazzaville Plage, il remonte jusqu'à l'antique sagesse des Grecs en citant en exergue une maxime de Socrate : 
« La vie que l'on ne soumet pas à l'examen ne vaut pas la peine d'être vécue. » regard étonné de Karen Ross, une scientifique pure et dure :

«Il ouvrit la porte, tendit les bras et dit : "Bonjour, Amy".

Une énorme forme noire passa la porte en sautillant et lui tomba dans les bras. Ross fut surprise de la taille de l'animal. Elle imaginait quelque chose de plus petit et de plus mignon. Amy était aussi volumineuse qu'une femme adulte.

Amy embrassa Elliott sur la joue, de ses grosses lèvres, sa grosse tête noire paraissant énorme à côté de celle de l'homme [...].

"Amy est contente ce matin?" demanda-t-il.

Les doigts d'Amy remuèrent rapidement près de sa joue, comme si elle chassait les mouches. [...] et Ross réalisa qu'Amy parlait. Elle parlait avec une vitesse surprenante. »

51 Ainsi Amy est douée de sentiment, de langage, mais aussi, comme la suite le montrera, de la faculté de rêver et de porter des jugements: elle sait qu'elle est un singe, elle connaît ses propres limites comme celles des autres. Ainsi, ce ne peut être, selon elle, qu'un « homme humain idiot » le laborantin qui vient de la vexer doublement en disant d'elle «ce qu'il est vilain!», ignorant ainsi et son sexe et sa dignité. Quant aux mystérieux et monstrueux hominidés, mi-gorilles mi-hommes, ou plutôt ni l'un ni l'autre, qu'elle est chargée d'identifier, ce sont, dans son langage primaire, « des choses mauvaises ».

Conformément à son statut de personnage, Amy est en effet dotée d'un programme narratif spécifique. Il consiste à « servir d'interprète ou peut-être même d'ambassadeur à l'humanité, dans ses contacts avec les créatures sauvages ». Quand, sur le terrain, les ordinateurs tombent en panne, quand toute la science et la technologie des hommes (blancs) se révèlent impuissantes à identifier le chaînon manquant entre l'homme et l'animal, alors la dernière ressource qui reste, c'est le primate. Mais un primate domestique, "gentil», qui collabore avec les hommes contre les Autres, primates sauvages et méchants ${ }^{21}$. En tant qu'informateur privilégié de l'homme, Amy est la première eth(n)ologue des singes.

53 Congo est plutôt un thriller d'espionnage scientifique qu'un roman de science-fiction. L'action se passe en 1979, et la techno-science dont il est fait étalage correspond à celle de la fin de ce siècle. La fiction se tient dans les limites du vraisemblable scientifique. On retrouve dans le roman, inséré sous forme de fiches, le savoir éthologique 
actuellement disponible, tel qu'on peut le trouver par exemple dans deux ouvrages de vulgarisation parus tous deux en 1990.

Le titre du premier est déjà tout un programme de roman : Les Hominidés non identifiés des forêts d'Afrique. L'auteure, Jacqueline Roumeguère-Eberhardt (1990), situe son enquête, ou plutôt sa quête, dans la mouvance surréaliste. La préface est d'un certain B. Heuvelmans dont les titres ronflants (Docteur ès sciences zoologiques, président de l'International Society of Cryptozoology) sont censés donner une caution scientifique à ces divagations fantasmatiques sur le yéti africain. Le second est d'une veine moins littéraire. Destins de singe est écrit par deux universitaires, un primatologue et un vétérinaire. Deux chapitres, intitulés «La paix des gorilles» et «Les chasses du chimpanzé ", pourraient d'ailleurs dévoiler très précisément les tenants et les aboutissants primatologiques de nos deux romans, respectivement Congo et Brazzaville Plage. Comme si les personnages de fiction étaient issus directement d'objets d'étude scientifique.

Dans la logique de ce vraisemblable scientifique, les deux romans ont pour protagoniste le couple formé de l'observateur (homme) et de l'observé (singe) ; et, comme l'exige la production de l'intérêt romanesque, ce couple apparie des êtres de sexes opposés. Pour Congo, on a vu quel type de lien unissait Peter Elliot et Amy, « sa » gorille de laboratoire. Dans Brazzaville Plage, les sexes de l'homme et du singe sont disposés à l'inverse. Le prière d'insérer présente Hope Clearwater comme une «jeune, belle et savante éthologue ». Comme narratrice, elle présente, dans les toutes premières lignes de son récit, Clovis, le chimpanzé mâle qui est à la fois l'objet désintéressé de ses recherches et le sujet d'affects auquel elle sera amenée à réagir avec une sensibilité toute féminine :

«Je n'ai jamais éprouvé beaucoup d'amitié pour Clovis, il était bien trop stupide pour inspirer une réelle affection, mais il a toujours eu droit à une petite place dans mon cœur, largement - je pense - à cause de sa manière instinctive d'entourer de ses mains ses parties génitales dès qu'il était nerveux ou inquiet. Une attitude plutôt attachante, à mon sens, et qui témoignait d'une vulnérabilité naturelle tout à fait contraire à ses humeurs coutumières: arrogance bravache ou bien égocentrisme total et complet. »

Clovis fait partie d'une petite communauté de chimpanzés dits "sudistes " parce que, ayant fait sécession d'avec leurs congénères du Nord, ils se sont implantés au sud d'une rivière que les primatologues qui les observent in vivo ont plaisamment appelée le Danube. Mais, pour les singes, ce Danube n'est pas une plaisanterie, c'est une véritable frontière naturelle que des "patrouilles" de nordistes franchissent en silence pour attaquer par surprise leurs "frères" sudistes et les exterminer avec une cruauté extrême, savante, bref, digne de celle qui semblait être, jusqu'à cette découverte, l'apanage de l'homme.

57 Le dernier sudiste survivant s'appelle Conrad - un nom qui n'a sans doute rien de fortuit... Sa mort atroce marque la fin de la guerre (civile ?) entre les chimpanzés ; elle marque aussi la fin du roman. Hope y assiste, impuissante à arrêter le massacre, mais « contente d'avoir été là pour mettre fin aux souffrances de Conrad », pour lui donner, comme on dit - si justement en l'occurrence - le coup de grâce :

«Je traversai le ruisseau en pataugeant pour chercher Conrad. Je le découvris, tordu et ensanglanté, sous les buissons de mesquinhos. Sa main droite avait été arrachée à hauteur du poignet et il brandit son moignon vers moi dans une parodie d'agression. Sa figure était rouge et pulpeuse, labourée par les ongles et les poings de Darius. Mais ses yeux bruns me regardaient toujours aussi fixement. Accusant? Plaidant? Hostiles? Stupéfaits? 
Je me mis à quatre pattes derrière lui pour qu'il ne me voie pas et je tirai à bout portant dans son crâne. »

Par cette intervention littéralement sympathique - qu'on ose dire « humanitaire » bien qu'elle s'applique ici à un animal et que trop souvent, dans les médias, le terme paraisse s'appliquer à des hommes supposés souffrir comme des bêtes -, la narratrice abandonne la réserve et la neutralité propres au scientifique pour finalement abolir dans la mort la distance qui la séparait de l'animal. Se mettant ainsi « à quatre pattes ", elle est enfin apte à le comprendre.

Ce rapprochement se trouve d'ailleurs esquissé quelques paragraphes plus haut, lorsqu'elle essaie de repousser les assaillants :

«"PARTEZ! leur criai-je, en braquant mon revolver. FILEZ ! FILEZ”! Mais qu'était un revolver pour eux, Homo Troglodytes? Et qu'étais-je, moi ? Juste un autre étrange singe bipède, paradant, bruyant, menaçant. "

60 Le passage laisse transparaître l'ambition allégorique du roman. Cette histoire de singes pourrait être une fable ou un conte philosophique sur l'homme du $\mathrm{xx}^{\mathrm{e}}$ siècle, qui a inventé aussi bien la compassion « humanitaire » que la violence " génocidaire ».

1 En guise d'intermède, on voudrait accorder au Congo lui-même une sorte de droit de réponse à tout ce romanesque anthropopithécologique produit dans le Nord.

2 Tout d'abord, en réponse à Brazzaville Plage lu ici comme un roman de la décivilisation, il faut dire que le renversement de l'homme en animal est un topos qui n'est pas propre à la littérature humaniste occidentale. On peut le rencontrer également dans une fable authentiquement congolaise, celle-là, puisqu'elle apparaît dans un roman de Sony Labou Tansi (1983) intitulé - de façon très significative pour notre propos - L'AntéPeuple:

"Comme disait la légende, les singes étaient des membres d'une tribu qui, des milliers d'années auparavant, avaient fui les impôts sous un gouvernement impitoyable. Ils avaient demandé à leurs ancêtres d'intervenir. Les ancêtres, pour la préserver du mal, transformèrent toute la tribu en singes. »

63 D'après l'auteur lui-même, cette histoire se racontait dans le clan de son père. De fait, on trouve dans un récit de voyage datant $\mathrm{du} \mathrm{XVII}^{\mathrm{e}}$ siècle un écho de cette « légende » sur les singes rapportée sous cette forme :

«La plupart des Nègres croient que c'est une Nation étrangère, qui s'est venue peupler dans leur Païs, et qu'ils ne parlent point de peur de travailler. »

À l'adresse, enfin, de Congo, il faut mentionner la réplique cinglante et immédiate de la presse congolaise. Celle-ci réagit non pas tant aux mots d'un livre qui se trouvait déjà en librairie depuis quelques années qu'aux images du film adapté du roman par John Marshall en 1995. Il y va en effet de l'image du Congo en tant qu'État-nation moderne et civilisé. Un premier article ${ }^{22}$ est ainsi résumé à la Une du doyen de la presse locale, un hebdomadaire d'obédience chrétienne qui jouit justement d'une grande considération :

«Aux États-Unis ${ }^{23}$, les Congolais sont "considérés" comme des gorilles tueurs. Histoire d'un film qui nous ridiculise : "Congo". »

Même écho indigné dans un second article ${ }^{24}$ publié dans l'hebdomadaire satirique le plus lu à Brazzaville :

«Il est aussi surprenant qu'agaçant que le Congo, en tant que triple concept géographique, historique et politique ne donne lieu dans l'imaginaire du réalisateur américain John Marshall - celui de Jurassik Park - qu'à des considérations sinon péjoratives, du moins bestiaires (j'allais dire bestiales). Mais de quoi se plaint-on si nous-mêmes ne faisons aucun effort pour améliorer notre image de marque aussi 
bien extérieure qu'intérieure ? [...] Sébastien Kamba ${ }^{25}$ doit penser à venger notre honneur frustré par cette identification nationale au symbole de King-Kong. » pleine d'humour de Hope qui se voit en Homo Troglodytes. Il est donc vrai qu'au roman des Occidentaux désespérés et décivilisés, Au cœur des ténèbres, répond, du cœur de ces mêmes ténèbres, un certain discours populaire ${ }^{26}$ autoraciste. Celui-ci expliquant celuilà, et inversement. Ainsi se recompose le «symbole » dans ce qu'on appelle à présent l'interculturel, pour ne pas dire interracial.

\section{Retour au Symbole, paradigme de la singerie}

72 La pratique du Symbole en usage dans l'école coloniale, on l'a vu, attache la singerie à la forêt congolaise et, par métonymie, à ses habitants sauvages. Cependant, le stéréotype de la sauvagerie primitive est à double tranchant, rien n'est plus aisé que de le retourner contre le prétendu civilisé ou "évolué", car la noblesse dont celui-ci se targue volontiers pourrait bien s'enraciner dans une singerie encore plus sauvage, ainsi que le notait récemment l'anthropologue congolais Joseph Tonda (2009: 133) dans une réflexion enracinée dans sa propre expérience :

«Voilà pourquoi l'humanisme civilisationnel qui présidait à notre scolarisation en français inventa un dispositif anti-exclusion d'une rare sauvagerie: le Symbole. Un mot terrible, un mot-Loi, un mot-objet. Le Symbole, c'était la terreur, la Loi et l'objet qui exhalait une puanteur épouvantable provenant d'un mélange de fèces, de cadavres de mille-pattes ou de crapauds enfermés dans une boîte de sardines ou de corned-beef, et qu'un coupable d'infraction à la Loi du Symbole devait porter au cou. L'infraction, c'était le patois». 
73 Le symbole a donc pour fonction de... symboliser clairement la séparation étanche entre la langue haute (le français) et la langue basse (le patois), séparation évidemment analogue à la ségrégation racialiste des locuteurs qu'elle reproduit sur le mode de l'abjection. Cependant, cette logique ségrégative a un effet pervers, elle est contrebalancée par l'imaginaire de la grégarité scolaire. La Loi du Symbole ne saurait en effet échapper à celle de l'Histoire : les écoliers coloniaux accédant peu à peu au statut de sujets francophones et francographes, la "distinction», qu'elle soit linguistique ou raciale, a tendance à perdre de sa fameuse clarté française. La langue française, à côté des "mots-objets violents", avait aussi des "mots-sujets », parmi lesquels J. Tonda se souvient notamment de "Madame». Or, "ce titre de haute noblesse », initialement et symboliquement réservé à la Dame blanche et opposé aux «bordelles » noires, va finir lui-même par être subverti à la fois par l'usage grotesque d'en bas et la politique d'assimilation d'en haut - celle-ci ouvrant la voie qui mène au « paradigme de la singerie » et ce jusqu'à présent :

«La subversion de la noblesse de Madame se réalisa cependant sous la forme de l'hérésie anticoloniale quand l'indépendance et ses suites firent surgir sur la scène des Madames qui n'étaient plus "Bana Poto" des enfants de Poto ou Mputu, déformation de Portugal! Cette hérésie fut sanctionnée par un grand éclat de rires paysans, ouvriers, écoliers, quand tel commandant noir, tel contremaître, tel instituteur, tel infirmier parlaient de leurs épouses en terme de madame! Le monde, décidément, avait changé, car il y avait désormais des Madames noires singeant les vraies [...]. Ce n'est pas seulement le grotesque de la singerie qui est ici en jeu. Ce qui est terrible, dans cette histoire, c'est que la singerie en question évoque des images de la guenon noire imitant la noble bourgeoise blanche. Le paradigme de la singerie était et est encore de nos jours un paradigme raciste en Afrique, comme l'atteste cette jeune Française qui, dans son exposé de soutenance de thèse dont je présidais récemment le jury quelque part en France, évoquait les Noirs qui singent les Blancs dans leur manière de s'habiller, de parler [...]. Je ne pus m'empêcher de penser à ces footballeurs africains à qui certains spectateurs français rappellent leur supposée nature véritable en singeant les singes dans leurs cris [...]».(Tonda $2009: 136)$

74 Ainsi se retourne la singerie, arme à double tranchant comme l'ironie. Le spectateur devient ici l'acteur de sa propre singerie, dont on soupçonne que les cris révèlent la «véritable nature » simiesque. Mais le plus remarquable dans cette affaire, c'est que le soupçon de singerie généralisée porte sur le langage en tant que critère crucial « d'hominisation du nègre » ou d'humanisation du singe. Et si la langue française, telle qu'elle s'impose dans le « Symbole », n'était elle-même qu'une imitation?

\section{Au Congo, berceau de la francophonie}

Que l'institution de la langue française au Congo rejoue la scène primitive de la singerie langagière, c'est précisément ce que donne à entendre un homme de lettres français voyageant en Afrique dans l'immédiat après-guerre pour délivrer le message de la nouvelle « Alliance française » à l'adresse de ses « chers Noirs » :

« Et, vous connaissant, nous avons trouvé naturel et logique de vous inviter à entrer dans la grande famille française comme nous sommes entrés jadis dans la grande famille gréco-latine ».(Bedel $1950: 97)$

76 L'invitation revient à proposer, sinon imposer, un contrat linguistique fondé sur l'antique matrice de la translatio studiorum: «Imitez-nous donc, Français qui avons imité nos ancêtres les Gaulois qui ont imité les Romains qui ont imité les Grecs qui 
[...].» Cependant, avant d'en venir aux «preuves» avancées à l'appui du mythe fondateur ${ }^{27}$, il convient de situer le contexte de ce discours, de voir en quoi il peut être considéré, après celui du grand homme de la France libre, comme le second discours de Brazzaville.

Maurice Bedel, auteur de plus d'une vingtaine de romans et essais, se définit comme un «missionnaire culturel», chargé de "dix siècles de pensée française ». En 1948, il s'envole pour un "voyage de conférences pour l'Alliance française, qui va [le] mener par l'Afrique noire jusqu'à Madagascar et aux Mascareignes", soit un "tourbillon de cent et quelques jours » (p. 251). Son journal de voyage paraît sous le titre de Tropiques noirs en 1950, soit quelques années seulement après le fameux discours de Brazzaville prononcé par le Général de Gaulle, le 30 janvier 1944. Faisons l'hypothèse qu'il en est la version culturelle, comme nous invite à le faire une des rares - et par là significatives - allusions politiques: "De là nous nous rendons dans une proche maison où m'accueille la fondatrice de la Maison de France qui, pendant la dernière guerre, soutint magnifiquement le mouvement gaulliste. Échange de propos, effusions sous le regard du Général de Gaulle accroché au mur en image photographique » (p. 233). Un autre indice que ce voyage se situe bien dans le prolongement politique et idéologique de l'Union française peut être trouvé dans le lieu où l'auteur choisit de transcrire le message qu'il est venu délivrer à l'Afrique : Brazzaville.

En effet, toutes les conférences qu'il a prononcées à chaque étape de sa mission mais dont il a évité jusqu'ici de nous donner la teneur sont condensées en un seul discours type, et ce discours est finalement livré au lecteur à l'étape de la capitale de l'Afrique équatoriale française. La "pseudo-paralipse » ou « ellipse latérale », est comblée par un discours pseudo-itératif ${ }^{28}$ soigneusement mis en scène.

La scène se passe au bord du Pool dans « un village de pêcheurs »; un décor statique et idyllique qui a l'avantage de fixer le public à qui le discours est adressé dans une posture a priori muette : «Ici, parmi ces cases batéké où les enfants et les femmes du village vont et viennent autour de moi, où les hommes appliqués réparent les mailles de leurs filets [...] », « Ici », par conséquent, l'orateur est seul en scène et nul ne saurait parler ni lui répondre, sinon les « évolués » qui sont les véritables interlocuteurs; mais justement ils ne sont pas ici, comme l'indique la suite de la phrase: " [...] j'aime à me rappeler tant et tant de palabres que j'ai eues avec les Noirs depuis des semaines. » On le voit, le discours est proféré, remémoré dans le silence du for intérieur; c'est un soliloque qui n'aura d'existence réelle que sur la page écrite dont les destinataires ultimes sont évidemment les lecteurs métropolitains. Ce dispositif énonciatif met donc en place un jeu complexe de triple adresse : ici, les villageois présents qui vaquent à leurs occupations traditionnelles, dont le silence affairé représente l'Afrique idyllique sous la colonisation française; là, les "évolués » dont la palabre est africaine et la langue, française ; là-bas, enfin, les Français de France qui possèdent pleinement le don de la langue et de la lecture.

Le lieu choisi pour le discours n'est pas moins symbolique du temps du voyage, car si notre missionnaire "s'attarde, désespéré à la pensée de les quitter demain, auprès des Noirs de la France africaine ", c'est parce que son voyage le conduira sur des territoires non français. Brazzaville est une ville doublement «capitale » en ce qu'elle est aussi bien une ville frontière dans la géographie coloniale qu'une étape stratégique qui marque une clausule dans le discours/ parcours du voyageur ; à ce titre, elle marque la fin nostalgique du périple en Afrique française. De l'autre côté du fleuve commence une 
autre Afrique, francophone certes, mais de culture belge, voire flamande. Le fleuve n'est pas un accident naturel mais une frontière culturelle; c'est un fossé que Maurice Bedel n'aura de cesse de creuser pour mettre en relief la spécificité de l'entreprise coloniale proprement française.

Un lieu idéal et un moment critique sont donc réunis pour faire le point et récapituler par l'écriture les grandes lignes du nouveau discours de la francophonie française :

«Chers Noirs, vous dont le cœur rouge est du même rouge que le mien, vous dont le cerveau dessine sous le crâne les mêmes dessins que le mien, je m'adresse à vous comme un frère à ses frères.

Je sais le tourment de votre esprit, je sais que vous eussiez préféré vous passer de nous dans l'organisation de votre indépendance sur le modèle des démocraties d'Europe et d'Amérique. Mais, vous, il faut que vous sachiez qu'une telle organisation n'est pas viable en ce moment sans notre conseil et notre appui.

Suivez-moi dans les preuves que je vais vous donner.

Il y a deux mille ans que nous nous sommes trouvés, nous, Français, vis-à-vis des Romains dans la même situation où vous, Noirs, vous vous trouvez vis-à-vis de nous. Les Romains, après avoir occupé nos terres et nous avoir imposé une administration en tous points semblable à celle que nous vous imposons, nous ont peu à peu donné le goût de vivre à leur façon, de penser et d'agir comme eux, de nous vêtir comme eux et de nous exprimer dans un drôle de sabir imité de leur langue, dans une sorte de petit-latin qui est devenu la langue de Bossuet.

Entre les Romains et nous s'était créée une symbiose.

Savez-vous ce que c'est qu'une symbiose? C'est entre deux êtres de famille différente une association telle que l'un ne puisse se passer de l'autre pour vivre: ainsi ces lichens [...] ».(Bedel 1950 : 96)

La suite du discours file la métaphore de la symbiose, dont on sait l'usage qu'en fera bientôt le poète L. S. Senghor, un des fondateurs officiels de la Francophonie. Mais cette analogie ne vient ici que pour persuader par l'image naturelle, pour renforcer l'argumentation par homologie qui est censée avoir déjà convaincu par la preuve historique. Le Noir est au Français ce que le Gaulois était au Romain : un colonisé dont le colonisateur attend qu'il se soumette à la Loi du Symbole - variante dure, sur le plan de la faune, de ce qu'est la symbiose pour la flore. D'un côté, le « petit-latin (pidgin des Gaulois) ${ }^{29}$ est au français classique ce que le petit-nègre est au parler scolaire destiné à «évoluer » en francophone. Et de l'autre, grâce à la symbiose civilisatrice, de même que le Gaulois est devenu le Gallo-Romain et enfin le Français de souche, de même le nègre est appelé à devenir un Noir francophone, autrement dit, de l'autre côté du fleuve, un «évolué ».

Ainsi passe-t-on de la France africaine à l'Afrique française, par ce tour de passe-passe qu'est la singerie originelle consistant à imiter l'idiome du vainqueur. C'est qu'une fois passé au Congo belge, notre pèlerin de la Francophonie laisse entrevoir le motif de la singerie. Il est vrai que plongé au cœur des ténèbres belges, où il a «de plus en plus l'impression que les Belges continuent à tenir les Noirs pour des Nègres ${ }^{30} »$, le voyageur humaniste laisse entendre que ceux-ci semblent n'avoir pas atteint le stade ultime de l'hominisation: "Difficile de rencontrer leur regard, encore plus de les entendre rire. Comme ils me sont étrangers! Comme je leur suis transparent!»En fait cette étrangeté des nègres colonisés, il l'impute implicitement au colon belge qui aurait dû suivre l'exemple que la France donne de l'autre côté du fleuve ${ }^{31}$; on sent entre les lignes que reste vivace la concurrence des colons inaugurée par la vieille rivalité entre les «pionniers », Stanley et Brazza. Laquelle s'enracine sans doute au plus profond du 
mythe du Barbare nordique (Germain ou Belge) opposé au Gaulois résistant ou Francien civilisé.

Tout au long de son périple au Congo belge, le Français ne peut s'empêcher de s'affliger au spectacle du Noir travaillant au bureau ou à l'usine, singeant le Blanc coupable de chercher à le "belgiciser ", autrement dit à faire du bon sauvage un nouveau barbare, moderne. Ce n'est que dans le cadre apaisé d'un éden tropical qu'on peut voir les vrais singes s'introduire dans le récit de voyage pour faire la leçon aux faux singes que sont devenus les « hominiens », noirs et blancs confondus :

« Dégagé des usines et des bureaux, me voilà séduit par les grâces déjà surannées du vieux Léopoldville, Léo II, comme on l'appelle, à l'écart du quartier neuf de Kalina. Réserve de palmiers et de leurs épiphytes dans une vallée idyllique, vieilles maisons coloniales à véranda et à jardin des tropiques, cimetière des pionniers enveloppé d'arbres congolais et où on lit sur les tombes les noms des compagnons de Stanley, des noms belges, allemands, suédois. On entend des chants d'oiseaux mêlés aux grondements des rapides; avec un peu de chance on voit des singes mener leur distraite curiosité autour des maisons des hommes, intrigués par l'odeur de viande grillée venue des cuisines et si éloignée du régime des hominiens. "Drôle d'idée", songent-ils en eux-mêmes, de s'empoisonner avec la chair des bêtes, nos sœurs à nous, quand les arbres offrent tant de fruits délicieux!».(Bedel $1950: 104$ )

Simple porte-parole de l'auteur, ce singe de papier, qui pense et parle en français, porte la critique d'inspiration rousseauiste de son maître opposé à la modernité cannibale. Son message est primitiviste, et sa formule favorite pourrait reprendre celle d'Oncle Vania, le singe anti-évolutionniste du fameux roman de Roy Lewis (1990), «back to the trees! $\aleph^{32}$ : ne suivez pas l'exemple des Belges d'ici, mais plutôt celui des Français de l'autre rive. Bref, il représente le « naturel » adapté à son environnement :

«Comment concilier la présence européenne tout en superflu, en recherché, en factice, avec l'humaine simplicité - j'aime ce mot - des naturels de ces lieux-ci ? [...] Ô Noirs de la forêt, Noirs des cases de palmes à l'ombre des parasoliers! Je souffre pour vous, et plus, j'en suis sûr, que vous n'en souffrez, de cette atteinte à vos naturelles habitudes. Quoi qu'en pensent les hygiénistes et les sociologues, il semble qu'il y ait dans ces alignements un manquement dû à la personne humaine ».(ibid. : 100-101)

Cependant, ailleurs qu'au Congo et même en Afrique, le singe est instrumentalisé dans une tout autre triangulation discursive. Arrivé à Madagascar où ses premiers mots sont pour citer la devise malgache «Mamy ny aina, la vie est douce» (ibid.: 177), notre pèlerin de l'Alliance française est confronté, non plus à la barbarie de la modernité belge, mais, au contraire, à la civilisation raffinée de l'ancienne royauté malgache qui a su résister à la conquête française - une mémoire ravivée par la récente « rébellion de la région d'Anosibé », quasiment passée sous silence dans son récit, mais que lui rappelle sans doute trop insolemment ce monument qui semble singer les ors et velours du pouvoir :

«Naturellement je ne coupe pas à la visite du palais de la Reine, de ses appartements style 1880, de son salon d'apparat tapissé d'un velouteux papier noir à fleurs d'or et meublé en son centre d'un canapé rond de velours grenat "que visaient les canons français du général Duchesne", me dit le jeune poète Flavien Ranaivo qui m'accompagne. Je n'aime pas le climat de nécropole des musées ; l'air m'y est irrespirable. Je bouscule la visite, j'avale sans reprendre souffle les trônes royaux et les filanzanes, les parasols rouges (couleur de la souveraineté) et les couronnes dorées, et je file vers Tsimbazaza, vers les jardins de la Recherche scientifique où je retrouverai le vivant Madagascar, le Madagascar des plantes et des bêtes ».(ibid. : 181-182) 
Dans ce paradis retrouvé, se renoue le pacte avec la nature authentique, incarnée ici par l'espèce endémique des lémuriens, des proto-singes qui passent aux yeux du touriste fasciné pour des animaux plus proches du genre hominien que les singes d'Afrique :

« Ma première visite est pour les lémurs. Je dois bien cela à ces chers petits animaux qui sont proches parents de l'homme et qui sont chez eux à Madagascar où leurs espèces sont si nombreuses qu'il est arrivé qu'on appelât Madagascar la Lémurie. [...] Mais j'aime à croire que cet étrange regard où il y a de l'étonnement et de la crainte est celui d'un être qui se trouve en présence d'un autre être dont il se sent proche parent sans bien saisir d'où vient la parenté. Pour moi, je me sens plus près d'eux que des cynocéphales ou même des chimpanzés. Qu'ils aient le museau pointu ou des dents de rongeurs, que les plus grands d'entre eux, les makis, ne dépassent pas la taille d'un chat (sans tenir compte de leur longue queue à fourrure annelée) peu m'importe : ce qui m'incline à les chérir c'est un quelque chose de tendrement inquiet, de peureusement affectueux que je n'ai jamais vu aux singes. Ils cherchent la caresse et craignent la semonce, de quoi le singe se moque bien ».(ibid. : 182-183)

En Lémurie ${ }^{33}$, ces "lémurs", selon l'appellation archaïsante empruntée aux Latins, constituent les vrais "naturels» du lieu; et ils sont doués des qualités natives (tendresse, affectivité, peur) que le touriste colonial (sous-) entend retrouver chez les naturels humains du même lieu, et qui font de l'indigène le sujet idéal de la colonisation française : la docilité. La comparaison des bons petits lémuriens avec les mauvais grands singes pourrait donc en cacher une autre, dont notre humaniste si féru de « la personne humaine» (Bedel 1948) ne saurait nommer le fondement raciologique. Masquant et dévoilant à la fois cet agenda caché, le raisonnement par homologie du parc zoologique de Tsimbazaza se révèle l'envers obscur de celui en discours de Brazzaville $^{34}$ : le lémurien est au singe ce que le Malgache est au Noir - soit, dans sa version raciste, le lémurien est à l'indigène de la Grande île ce que le singe est au Noir d'Afrique : un prototype d'humanité autochtone. Avec la leçon cachée (subliminale ?) que le Continental devrait prendre l'Insulaire pour modèle, accepter docilement la "symbiose" et le «symbole», et non leur résister. Car l'Évolution (naturelle, biologique...) est supposée irrésistible, à l'instar du Progrès (culturel, linguistique...) dont elle est d'ailleurs devenue en ce $\mathrm{xx}^{\mathrm{e}}$ siècle, le modèle stéréotypé de validation et de véridiction scientifique.

\section{Derniers avatars...}

89 Quelque soixante ans après M. Bedel, Patrick Besson (2009), homme de lettres français, chroniqueur et polémiste bien connu, séjourne quelque temps au Congo ; il en sort un roman fleuve, Mais le fleuve tuera l'homme blanc, qui fait de Brazzaville « le théâtre d'un thriller politique provocant, entre violence, sexe et corruption $»^{35}$ censé valoir, aux yeux de la critique, pour le portrait craché de toute l'Afrique noire post-Rwanda. Dans le concert de louanges que l'éditeur reprend en guise de publicités ${ }^{36}$, il est même cité à l'égal de l'auteur du Coeur des ténèbres: "Ce Besson-là frappe comme Joseph Conrad.» Comme si on ne pouvait parler du Congo sans remonter à Conrad... Ainsi pointe le museau de la singerie au Congo, mais en habits neufs. Il n'y a pas de forêt profonde dans la Brazzaville de ce " coco au Congo $»^{37}$, ni gorille ni chimpanzés ${ }^{38}$. Pourtant, s'il n'est visible nulle part, le paradigme de la singerie est présent partout. Ce qui en fait le lit, ce sont toutes ces incessantes saillies du récit et sorties du temps, où se formule, presque à chaque page et chez tous les personnages, la sentence du moraliste hanté par 
le génie perdu, la vérité générale réduite à la portion ethnique (le Bantou / la Tutsie / le Congolais / les Kongo(lais) / le Blanc / les Africains, etc. Autant de clichés essentialistes et dualistes qui s'inscrivent d'abord sur le souvenir-écran nostalgique de la France Libre : « Avant d'être une capitale bougonne et désorganisée, [...] Brazzaville a été le centre de l'Afrique francophone. De Gaulle fut l'homme de Brazzaville avant d'être de Colombey. [...] Brazza est autant que Londres le berceau de la Résistance et du gaullisme. » Dès lors, le démon de l'analogie peut se donner libre cours dans le champ ouvert aux homologies géopolitiques :

«Le Congo était une URSS en petit et en noir, constituant la frontière australe du socialisme. Le fleuve en mur de Berlin poreux, vu le nombre de gens qui, menacés sur une rive, se réfugiaient sur l'autre. Au contraire de l'Europe, le capitalisme se trouvait à l'est et le communisme à l'ouest. Les Congolais de l'Ouest - les Cocongolais, les appelaient les gars de la Polex - enviaient-ils les Congolais de l'Est surnommés, à la même Polex, les Congolestes, comme les Allemands de RDA ceux de RFA ».(Besson 2009 : 164)

90 Et aux raccourcis historiques ou géographiques :

«On reconstituera le royaume Kongo, démembré autrefois par les Portugais, les Français, les Belges, les Allemands. Dans chaque région il y aura un gouverneur tutsi. Nous sommes destinés de toute éternité à gouverner. [...] vers l'empire tutsi, le Grand Empire tutsi ».(ibid. : 147)

91 Voire aux étymologies controuvées, comme pour ces enfants des rues, déjà assimilés à des « enfants sorciers " (frisson exotique garanti), dont le nom "Chégués » viendrait de la "contraction de Che Guevara, héros congolais", alors que, dans la conscience populaire, le Shege serait le calque lingala de Schengen (Biaya 2001:20) ${ }^{39}$. Il faut bien que les enfants singent le père, ce héros étranger et blanc...

Cependant, l'enjeu de la singerie au Congo - «Je dirais passion. Obsession. Fascination" - se révèle surtout à propos d'un "génie nommé Sony Labou Tansi »" auquel P. Besson consacre presque un chapitre entier par la voix de Bernard, un personnage ami et biographe de "l'écrivain bakongo $»^{41}$. Reprenant la polémique sur l'authenticité des romans ${ }^{42}$, Bernard soutient qu'ils ont perdu leur foncière originalité parce qu'ils auraient été, en aval, « revus, corrigés, nettoyés et retaillés par le personnel littéraire français», au point qu'il «y a deux œuvres de Sony Labou Tansi : celle qui arrive chez l'éditeur et celle qui ressort " (ibid. : 229). Mais c'est en amont, justement à l'autre bout de cette "chaîne ", que la singerie est la plus flagrante, affichée ici sous le régime du plagiat généralisé :

«Sony pompe Marquez, qui a pompé Faulkner, qui a pompé Joyce, qui a pompé Homère. Mais Homère n'a pompé personne et personne ne pompe Sony. Ils sont le début et la fin de la chaîne du génie littéraire ».(ibid. : 230)

Ainsi, seule la théorie du génie ${ }^{43}$ paraît pouvoir arrêter la course folle vers l'origine, la régression infinie vers la source, vers le premier signe obscur du singe ou nègre originel. Elle en est le versant glorieux, lumineux. Le phare au cœur des ténèbres, entre hominisation du nègre et humanisation du singe. Génie littéraire ou singe plagiaire font bon ménage, « ayant enfin réalisé la synthèse des deux couleurs [noir/blanc] qui l'ont, qui m'ont toujours obsédé » (ibid. : 233).

À quoi Sony (1997 : 11), indocile et inclassable, a déjà répondu par avance :

« Un jour, il y en a qui diront : je l'ai influencé - ils seront nombreux - et voici ma réponse : à tous ceux-là qui croient qu'ils m'ont influencé je dirai : d'accord, vous m'avez influencé, mais je suis allé plus loin que vous, j'ai sauté plus haut que vous, 
accusez-moi de cela, pas d'autre chose - autrement, soyez fiers de m'avoir engendré : c'est votre droit, après tout. »

Cependant qu'à Brazzaville, là où Madame, inaltérable allégorie de la Langue française, peut continuer à se mirer devant sa chère afro-descendance francophone, le paradigme de la singerie ${ }^{44}$ n'en finit pas de produire des « répliques » sous influence, comme en cette admirable prosopopée académique :

«Moi, Dame française en terre congolaise, qui vous parle de mes sept siècles d'âge (car je suis bien plus vieille qu'on se l'imagine malgré ce visage de bonbon que je porte), n'ai jamais... jamais été autant comblée de joie. De joie et de bonheur de constater que, parmi les 49 filles que j'ai faites, lesquelles m'ont à leur tour donné 170 millions de mômes, vous les enfants, vous êtes de ceux qui me titillent le mieux, me taquinent le plus. Ça m'amuse. Chose rare, vous m'écoutez aussi. Ah, comme je suis contente de vos grands frères que sont Loutard et U Tamsi. Ceux-là ! Tout autour d'elle, les enfants se mirent à rire, aussitôt ramenés au silence. Prunelles grandement ouvertes, ils suivaient attentivement cette femme aussi vieille que leur grand-mère munukutuba, qui se disait leur grand-mère et s'exprimait dans une langue qui de loin ressemblait à la leur. Mais qui, et pourtant sonnait comme le français ! La vieille devait revenir de loin, et pour les enfants, c'était une occasion à ne pas manquer [...]. Ici, les enfants, je me sens chez moi. Je respire de l'air frais, cet air qui me rappelle les arômes des bois de Boulogne. Comment m'empêcher de vous le dire ? Oh mes petits enfants, comme vous êtes rigolos, très amusants. Sachez que votre grand-mère, laquelle a marié votre mère, sa fille, à Monsieur le Congo votre père, voici déjà bien des lustres, vous aime beaucoup. Et ce, malgré que sous vos langues, la pauvre vieille n'ait jamais été si heureuse que ça. Vous conviendrez avec moi que ça ferait pas plaisir à vos oncles Molière, Racine et Corneille s'ils l'apprenaient ! Mais que voulez-vous, ainsi va le monde. On a tous besoin un jour ou l'autre d'un peu de changement. Ça réconforte, ça enrichit. Car vous me faites moins de mal que je ne pense. Au contraire, je me sens enrichie de mille caresses tendres, émoustillantes. Ça fait rigoler! Ah les mots, ils ne sont pas ici que nous croyons là-bas; ils refusent de vieillir comme moi. Et alors, ça rajeunit en changeant de sens. Ça joue au porteur de masques, les mots... $»^{45}$.

\section{BIBLIOGRAPHIE}

BALANDIER, G., 1995 [1955], Sociologie actuelle de l'Afrique noire : dynamique des changements sociaux en Afrique centrale, Paris, PUF.

DE BALEINE, P., 1993 Voyage espiègle et romanesque dans le petit train du Congo, Paris, Presses Pocket.

BEDEL, M., 1948 Destin de la personne humaine, Paris, Bader-Dufour.

-, 1950 Tropiques noirs, Paris, Hachette.

BESSON, P., 2009 Mais le fleuve tuera l'homme blanc, Paris, Fayard.

BIAYA, T. K., 2001 « Jeunes et culture de la rue en Afrique urbaine (Addis-Abeba, Dakar et

Kinshasa) », Politique africaine, numéro spécial « Enfants, jeunes et politiques », $80:$ 12-31.

BLACHÈRE, J.-C., 1993 Négritures. Les écrivains d'Afrique noire et la langue française, Paris, L'Harmattan. 
BOYD, W., 1991 Brazzaville Plage, Paris, Éditions du Seuil.

CERQUIGLINI, B., 2007 Une langue orpheline, Paris, Éditions de Minuit.

CONSTANT, P., 1989 White Spirit, Paris, Gallimard.

CRICHTON, M., 1990 Congo, Paris, Christian Bourgois (« 10/18 »).

DESPORTES, C. \& THIERRY, B., 1990 Destins de singe, Paris, Acropole.

FRANCESCHI, P., 1991 Au Congo jusqu'au cou : expédition Babinga-Pongo, juin-octobre 1975, ou l'aventure initiatique et extrême de quatre Français de 20 ans chez les Pygmées de la forêt équatoriale, Rennes, Ouest-France Éditions («Jusqu'au bout du voyage »).

GENETTE, G., 1972 Figures III, Paris, Éditions du Seuil.

GIDE, A., 1914 Les Caves du Vatican, Paris, Éditions de la Nouvelle Revue Française.

GREENE, G., 1960 La Saison des pluies, Paris, Presses Pocket.

GUEVARA, E. Che, 2009 Journal du Congo, (trad. par René Solis), Paris, Mille et une nuits.

HAGERFORS, L., 1990 Les Baleines du Lac Tanganyika, Paris, Flammarion.

HALEN, P., 1993 Le Petit Belge avait vu grand. Une littérature coloniale, Bruxelles, Éditions Labor/ Archives et Musée de la littérature.

HOMBERT, J.-M. \& PERROIS, L. (dir.), 2007 Cour d'Afrique. Gorilles, cannibales et Pygmées dans le Gabon de Paul de Chaillu, Paris, CNRS Éditions.

JAСОВ, C., 1991 « Aux confins de l'humanité : peuples et paysages africains dans le Périple

d'Hannon », Cahiers d'Études africaines, XXXI (1-2), 121-122 : 9-27.

KING, S., 1994 Le Singe, Paris, Librio.

LABOU TANSI, S., 1983 L'Anté-Peuple, Paris, Éditions du Seuil., 1997 L'autre monde, écrits inédits, Paris, Revue Noire.

LE BIHAN, A., 1993 Retour de Lémurie, Paris, Françoise Bourin.

LE GOFF, J., 1980 « Le désert-forêt dans l’Occident médiéval », Traverses, 19 : 23-33.

LESTRADE, P., 1993 La Montagne des Singes, Paris, Phébus.

LEWIS, R., 1990 Pourquoi j'ai mangé mon père (trad. et préf. par Vercors), Arles, Actes Sud (« Babel »).

MARTIN-GRANEL, N., 1995 « Discours de la honte », Cahiers d'Études africaines, XXV (4), 140 : 739-796.

-, 2001 « Monkey Business in the Congo », in M. PALMBERG (ed.), Encounter Images in the Meetings between Africa and Europe, Uppsala, Nordiska Afrikainstitutet : 206-220.

-, À paraître, « Le Congo concept », Actes du colloque APELA Littératures africaines et territoires, Bayonne, septembre 2009.

MESCHONNIC, H., 1997 De la langue française : essai sur une clarté obscure, Paris, Hachette.

MIANO, L., 2009 Les Aubes écarlates, Paris, Plon.

O'HANLON, R., 1997 Congo Journey (trad. par Jacques Chabert sous le titre O'Hanlon au Congo), Paris, Flammarion (« Gulliver »).

QUAGHEBEUR, M., 1992 « Des textes sous le boisseau », Papier blanc, encre noire, Bruxelles, Labor : VII-XCIV. 
RIO, M., 1985 Les Jungles pensives, Paris, Balland.

ROUMEGUÈRE-EBERHARDT, J., 1990 Les Hominidés non identifiés des forêts d'Afrique, Paris, Robert Laffont, (« Dossiers X »).

von stroheim, E., 2001 [1956] Poto Poto (préf. de Blaise Cendrars, trad. par Renée Nitschke), Paris, Pygmalion.

TONDA, J., 2002 La Guérison divine en Afrique centrale (Congo, Gabon), Paris, Karthala.

,- 2009 « Mots-objets, mots-sujets, mots-esprits », in Riveneuve Continents, 8 : 132-140.

WIJKMARK, C.-H., 1986 La Draisine, Arles, Actes Sud.

YENGO, P., À paraître, Le ventre et sa politique, Paris, Karthala.

\section{NOTES}

2. Un syndrome qui s'enracine dans la tradition proverbiale. Des égoïstes, on dit par exemple qu'ils « s'agrippent à leur poste comme un singe à leur fruit » (La Rue meurt, 63, 6/01/1995). Dans ce journal satirique, la même image revient, mise au goût de l'actualité, appliquée aux vieux chefs d'État du monde: "Ces derniers temps nos "ancêtres" au pouvoir ont tous la mauvaise malchance de passer sur le billard [...]. Alors, mes frères, prions fort pour ces grands pères tout en leur demandant de renoncer à jouer au singe qui meurt avec son fruit » (La Rue meurt, 151, 19/09/1996).

3. Au cours d'une série d'entretiens avec le journaliste Apollinaire Singhou Basseha sur les ondes de La Voix de la Révolution congolaise.

4. Sur cette identité « équivoque », voir Georges BALANDIER (1995: 289).

5. Pour Joseph TONDA (2002: 105), ce «néologisme congolais formé à partir du français "mon singe", c'est-à-dire "l'indigène" approprié par le Blanc sous le terme animalisant de "mon singe" » constitue la face cachée de "l'évolué ", lequel « réunit, à travers les critères de sa réalisation, en particulier la "maîtrise" de la langue française, de l'écriture et des "manières" françaises ou européennes, toutes les caractéristiques de l'identité valorisée mais jamais suffisante aux yeux de la puissance blanche qui la produit pour prétendre l'égaler. Il s'agit donc d'une identité précaire, parce que dépendante de la puissance coloniale qui l'institue tout en la dévalorisant. À cette identité "équivoque" s'opposent celles dont la forme extrême fut dans les deux Congos, celle du mossendzi ».

6. Un discours critique souvent mêlé d'autodérision, dont on pourrait trouver un exemple probant dans le récent roman de Léonora MIANo (2009: 138): «On avait pénétré dans une aire d'hybridité. Cependant, les Continentaux [Africains] ne parvenaient pas à inscrire leur expérience dans la globalité de l'aventure humaine. Ils ne pouvaient dépasser les représentations négatives qu'on avait eues d'eux. Encore emmurés dans ces vieilles conceptions, ils ne parlaient jamais de ce qu'ils pensaient d'eux-mêmes, mais de la manière dont ils avaient été considérés : humains inaboutis, singes un tantinet plus évolués que les autres. Il était donc inutile de leur dire aujourd'hui que tout cela était loin, que c'était fini, que maintenant on les aimait, que black était fashionable, qu'on savait qu'ils pouvaient travailler à la Nasa et écrire des livres [...]. On les avait exclus du genre humain ». 
7. «Bon Dieu est raciste », La Rue meurt, 148, 29/08/1996, p. 12.

8. Sans doute faut-il voir dans cette mythologie noire l'origine du titre du roman de Joseph Conrad, Au cœur des ténèbres, et peut-être même du Voyage au bout de la nuit de Céline, au moins pour son épisode africain.

9. À propos de romanesque cinématographique, il faudrait signaler la transposition de Au cour des ténèbres par Coppola dans Apocalypse Now, ainsi que Poto-poto, un roman écrit comme un scénario de film par Erich von STROHEIM (2001) - des œuvres qui exploitent l'image de la forêt comme un désert et enfer vert.

10. Dernièrement le journal Libération (3/4-10-2009) lui consacrait un hommage appuyé sous la plume de son directeur de rédaction qui le pose en nouvel héros des Lumières, tout à la fois "explorateur, ethnologue et romancier». Sous le titre de "Franceschi mène sa barque ", le long "portrait ", illustré de photos exotiques et d'un primitivisme étonnamment anachronique, dépeint ce fils de la Corse profonde (et gaulliste !) comme «missionné par le ministère de l'Écologie, comme Bougainville en son temps » repartant «à bord de sa Boudeuse » vers de nouvelles aventures humanistes voire humanitaires. Celle du Congo est retracée comme une légende: étudiant, déjà il " préfère Conrad à ses polycopiés »; puis « un jour, il entend qu'une portion d'Afrique, confetti sur la carte du continent, reste inviolée »; enfin vient la sanction du terrain : «La leçon est rude. L'endroit est un enfer sur Terre, une jungle invivable, regorgeant de bêtes venimeuses, de plantes empoisonnées et de marécages, où jamais un Pygmée n'aurait eu l'absurde idée de s'installer. »

11. Je remercie Florence Bernault de m'avoir signalé le branchement croisé, par le motif excrémentiel, entre ces deux stéréotypes de singerie - dans la forêt et l'école toutes deux dites "primaires ", terrains glissants où l'explorateur ou l'élève a vite fait de se retrouver dans la m... jusqu'au cou.

12. Le nom même est un viatique qui, transmis de voyageur en voyageur, suffit à conférer une aura mythique au voyage : «Conrad! Je frémis en lisant, sous la plume d'André Gide, qu'il a traversé à pied en 1890 ces mêmes montagnes qu'escalade mon train et qu'il décrit en leur intacte horreur dans son "Cœur des ténèbres" " (DE BALEINE $1993: 20)$.

13. Un stand entier lui a d'ailleurs été consacré au dernier Salon du livre de Paris, à l'initiative du ministère de la Culture et des Arts du Congo, des Dépêches de Brazzaville et de la Galerie Congo.

14. Voir Jean-Marie HOMBERT et Louis PERROIS (2007). Si l'éditeur, les signataires et la teneur même relèvent bien évidemment des sciences humaines les plus sérieuses, cet ouvrage, par ses titres et son habillage, paraît singer le "beau livre "; destiné à réhabiliter un personnage d'outsider dans la course à l'exploration des merveilles du Congo, il invite aussi à produire un intérêt quasi romanesque, ainsi qu'en témoigne la quatrième de couverture en sa péroraison: "L'heure est venue pour ce pionnier de l'Afrique, aussi important par ses travaux et ses découvertes que Brazza ou Livingstone, de rencontrer le grand public. Une équipe de chercheurs français et gabonais entreprend, dans cet ouvrage magnifiquement illustré, de rendre justice à ce personnage hors normes du XIX ${ }^{\mathrm{e}}$ siècle. »

15. Sur ce motif du singe compagnon obligé du voyage au Congo, voir Redmond O'HANLON (1997). Ce « Tarzan intello » en adopte un au cours de sa longue marche, à en croire la quatrième de couverture, "vers ce "cœur des ténèbres" que hantent les 
fantômes de Stanley, et de Conrad [...]. En bateau, d'abord, en pirogue, puis à pied. Et l'on dirait que tout ce qui pique, rampe, englue, dévore, empoisonne sur terre s'est donné rendez-vous sur son passage. Parviendra-t-il - et dans quel état - au lac Télé ? Y trouvera-t-il le fameux dinosaure? » Plus étonnant d'apprendre au détour d'une phrase que même un guérillero tel le Che en avait pris comme mascottes : «[...] et nous nous retrouvâmes sur la route, laissant derrière nous une innombrable quantité de choses : des livres, des papiers, de la nourriture et même deux petits singes que j'avais comme mascottes » (GUEVARA 2009 : 239).

16. Pour reprendre le nom (le roman de la "décivilisation») par lequel Pierre HALEN (1993) désigne ce sous-genre de la littérature coloniale dont les prototypes furent les romans « africains » de Conrad et de Céline.

17. Sur un prospectus d'une agence de voyages locale s'adressant à la clientèle expatriée de Brazzaville on peut lire: "Découvrez un éden pittoresque du Congo profond " ("Confort voyages », mai 1996).

18. Voir aussi le Voyage espiègle de DE BALEINE (1993). On y trouve, énoncés d'un ton paternaliste ou amusé, tous les motifs poncifs, depuis le gorille violeur de femmes blanches (le premier chapitre s'intitule "Gare aux gorilles») jusqu'aux chasses sauvages des braconniers qui déciment les « proches cousins » de l'homme, sans oublier le vol des bébés gorilles qui défraye la chronique des journaux locaux. Signalons que l'auteur de ce récit de voyage postcolonial collabore à Paris Match, un hebdomadaire dans lequel il a publié notamment une interview ("Faut-il se dégager de l'Afrique... », 1993) avec Bernard Lugan; celui-ci, déjà connu pour ses prises de position négationnistes, y défend la thèse cartiériste en s'appuyant sur le mot de Lyautey « Ils ne sont pas inférieurs, ils sont autres ».

19. Auxquels il faudrait ajouter celui du Suédois Carl-Henning wIJKMARK (1986). Son ironie réflexive, comparable à celle de son compatriote L. Hagerfors ou de M. Rio sur le même sujet simiesque, aurait mérité une analyse plus fouillée; contentons-nous ici de mentionner « le point de vue des éditeurs ", son accroche publicitaire en quatrième de couverture :

«Ces fascinantes tribulations de trois singes et d'un Jésuite commencent avec l'invention de la draisine à voile, un engin que l'ingénieur Dittel a mis au point pour circuler sur la voie ferrée dont il dirige la construction, au Congo belge, en 1914. De la brousse, en effet, l'insolite véhicule fait sortir trois singes si familiers que l'ingénieur les baptise (Paul, Mathilde, Jacob) et les prend à son service pour manœuvrer la draisine. Or, près du fleuve, un Jésuite en rupture de ban, adepte des théories évolutionnistes et grand lecteur de Darwin, s'intéresse lui aussi à ces anthropoïdes auxquels ne manque que la parole. »

20. Un cas intermédiaire pourrait être trouvé dans un autre roman "congolais ", de Pierre LESTRADE (1993). Des gorilles identifiés comme des individus et dotés d'un nom, y occupent un espace neutre entre l'homme et le singe, entre la forêt et le village : "Là où vit le gorille, dans des aires bien circonscrites telles qu'ici, il donne à la forêt, comment dirais-je, oui, qui n'est ni de l'animal ni de l'homme. Bien sûr, on vous dira qu'ils sont inoffensifs. Je dirais plutôt qu'ils sont imprévisibles, tout comme nous » (ibid. : 240). Par ailleurs, dans cette forêt "à la Stanley », il se passe des choses bizarres : «Ce gorille n'était-il pas devenu un mutant, doté de l'agressivité gratuite qui est le propre de l'homme?» 
21. On peut se demander s'il ne s'agit pas là d'un fantasme raciste typique d'une certaine Amérique. Ainsi, dans la nouvelle fantastique de Stephen KING (1994) qui a pour titre Le Singe, un simple jouet en peluche suffit à créer l'horreur. Pour l'adulte qu'est Hal, cet "objet maudit ", avec "son sourire sans âge», provoque "une sensation de dégoût presqu'instinctive ». Et ce dégoût est comparé par deux fois à celui que pourrait provoquer le premier habitant de la forêt : «Comme s'il venait de découvrir un Pygmée meurtrier "; "Il était vivant, se contorsionnant entre ses mains comme un pygmée répugnant [...]. »

22. "Hollywood se fait des milliards sur le dos des Congolais », La Semaine africaine, 2069 du 2/5/95, p. 1 et 9. Soulignons qu'il est exceptionnel pour ce journal qu'il mette à la Une un article de la rubrique culture et, qui plus est, illustré d'une photo (un bébé singe derrière des barreaux). Précisons enfin que l'auteur de l'article, Jérémie Dongala, réside aux États-Unis où il occupe de hautes fonctions dans un organisme international et qu'il est le frère de l'écrivain congolais, E. B. Dongala.

23. Où persistent, même dans la cité cosmopolite de New York et à l'ère supposée postraciale d'Obama, «les sombres desseins » de la singerie primitive : «Tout est parti d'un fait divers : en février, un chimpanzé qui avait manqué de tuer une femme a été abattu par la police. Le lendemain, le New York Post illustrait l'histoire par une caricature montrant deux policiers penchés sur le cadavre du primate, l'un deux disant : "Ils vont devoir trouver quelqu'un d'autre pour le plan de relance économique" " (Libération, 13/11/09).

24. «Congo fait du cinéma », La Rue meurt, 96 du 31/8/ 95, p. 4.

25. Le réalisateur congolais le plus célèbre dans son pays.

26. Au sens d'une opinion diffuse dans tout le champ social, apparaissant aussi bien dans un journal "populaire» - par exemple Le Vagabond, dans un article titré " Délinquance sénile »: " Nos pères sont beaucoup plus nos gorilles que nos parents " (6 juin 1993) - que dans un discours officiel au plus haut niveau. Ainsi, le 18 juillet 1996 le président de la République du Congo, Pascal Lissouba, en réponse au toast de son homologue français, Jacques Chirac, pouvait-il rendre hommage à de Gaulle comme "l'homme qui a permis l'hominisation du Nègre" (sic) sans faire sourciller ses adversaires politiques. Pas plus d'ailleurs que son public d'intellectuels conviés à relever le défi de la Renaissance d'une Afrique de l'âge d'or dépeinte par Dapper, lorsqu'il concluait son discours en ces termes: "Sinon, qu'on retourne au jardin zoologique!»

27. Pour une déconstruction critique de ce mythe du français latino-descendant, voir Bernard CERQUIGLINI (2007).

28. Pour une définition de ces termes, voir Gérard GENETTE $(1972$ : 93, 152).

29. C'est au Cameroun qu'est formulé le mythe de l'homologie historique: "Très intéressant entretien avec Brinon sur le parler "petit-nègre". Ici c'est le pidgin. Le pidgin tend à prendre la place des langues locales comme le "petit-latin" (pidgin des Gaulois) s'est substitué aux dialectes celtiques. Pour Brinon, il y aura bientôt en Afrique Noire une langue pidgin, contrefaçon du français, comme il y a en France une langue française, contrefaçon du latin » (BEDEL 1950 : 79).

30. Notons que cette phrase a été supprimée dans le volume de 1950. Dans la version publiée en 1949 dans une revue belge, elle faisait suite à la réflexion sur les habitants 
autochtones de Luluabourg, les Mongo: "Ils se sont soulevés il y a peu de temps; révolte de nègres plutôt que soulèvement de Noirs » (ibid. : 109).

31. "C'est que le Belge a transporté ici sa Belgique alors que le Français a tâché d'adapter sa France au décor et au climat de l'Afrique » (ibid. : 101).

32. Notons le tropisme congolais d'Oncle Vania : «En primate raisonnable, j'ai donc été voir un peu plus loin si je ne trouverais pas des aliments conformes à mon état. Au Congo pour tout dire. Il y a dans ce coin abondance de tout, pour tout le monde » (LEWIS $1990: 17)$.

33. Sur les positionnements récents de la francophonie malgache, voir les réflexions particulièrement lucides d'un ancien délégué général de l'Alliance française au début des années 1990, Adrien LE BIHAN (1993).

34. Que les deux discours sont connectés, on en voit le symptôme dans cette résurgence récente à Madagascar à propos de l'affaire des bienfaits de la colonisation : "Que serait Aimé Césaire, et autres chantres de la négritude, si son pays n'avait pas été colonisé ? Et si les Gaulois n'avaient pas été colonisés pendant près de 500 ans - oui cinq siècles ! par les Romains, peut-être n'auraient-ils jamais eu l'idée de faire, un jour, pareil ?» ("Éditorial », Les Nouvelles, 12 décembre 2005).

35. «Une Afrique à la machette " (Le Monde, 4 septembre 2009). Voir aussi « Voyage au bout de l'Afrique » (Le Journal du Dimanche, 13 septembre), «Ce roman a le souffle long, il en faut pour prendre l'Afrique noire à bras-le-corps » (Le Point, 27 août).

36. Le Monde, 16 octobre 2009.

37. Le Nouvel Observateur, 27 août 2009.

38. Sinon comme insulte dans "Tintin au Gabon", un éditorial qui vise à défendre par l'ironie l'album d'Hergé attaqué en justice pour racisme : « Haddock : Regardez, Tintin, comment ces primates traitent les bâtiments français! Tonnerre de Brest! Tintin: J'admets, capitaine, que cette violence n'est guère compréhensible. Les Gabonais oublient bien vite tout ce que la France a fait pour eux » (Le Point, 10 septembre 2009).

39. Voir Patrice YENGO (à paraître), rappelant le contexte : "Popularisé par le chanteur congolais Papa Wemba dans sa chanson "Kokokorobo", le terme Shege est devenu l'hyperonyme de l'enfant de la rue ", penche lui aussi pour cette seconde étymologie "plus répandue, qui le fait procéder du nom de Schengen que l'imaginaire urbain subsaharien assimile à la frontière européenne contre laquelle viennent s'échouer tous les jeunes préposés à l'émigration. Mais au-delà de l'échec que ce trope dissimule, cette appellation est devenue la revendication identitaire d'une certaine urbanité pour les jeunes déclassés de la mégapole kinoise qui s'affirme par la réappropriation imaginaire d'une culture globale combinée aux traits locaux où les formes de violence individuelle côtoient les violences martiales, dans un esthétisme païen et politique, où se donnent à voir des actes d'effronterie qui moquent le pouvoir central dans une sorte d'érotisme à l'imaginaire torturé. »

40. Jeune Afrique, 16-29 août 2009, pp. 170, 172.

41. Dont la lettre initiale $\mathrm{K}$, supposée plus originellement africaine, contamine la graphie de noms qui lui sont étrangers, comme Kokoye (ibid.: 320) ou Tchikaya (ibid.: 132). Singerie orthographique ? Sur le sens de la lettre $K$ comme signe cryptographique à la recherche du Kongo perdu, voir MARTIN-GRANEL (à paraitre). 
42. Pour une résurgence récente au Burkina Faso, voir «L'écrivain congolais Sony Labou Tansi a-t-il eu des nègres? ", sur le site de Rue89, 22 novembre 2008 :

<http://www.rue89.com/2008/11/22/lecrivain-congolais-sony-labou-tansi-a-t-il-eudes-negres>. Pour une mise en perspective historique de la question, voir Jean-Claude BLACHÈRE (1993).

43. Pour une critique du mythe de la clarté et du génie de la langue française, voir Henri MESCHONNIC (1997).

44. Pour une illustration du branchement récent de ce paradigme et du concept Congo sur la Toile, on peut lire, dans le portrait de Kamel Toe : «Ce Nordiste [qui] procède sur Internet à une relecture toute personnelle des classiques de la littérature ", cet extrait significatif: «Dom Juan, c'est un cynique, comme le nom de l'aut'babouin [Diogène cynocéphale ?], là, et en vérité, il est malhonnête à fond, c'est un mec malhonnête avec des couilles fat comme des pastèques du Congo » (Libération, 14 avril 2010).

45. Texte cité et envoyé par télégramme diplomatique avec le commentaire suivant: "Troisième prix du concours "Le français tel qu'on le parle au Congo" proposé par le CCF de Brazzaville à l'occasion des Journées de la francophonie 2002, ce texte écrit par un jeune Brazzavillois de 20 ans, étudiant à l'Université Marien Ngouabi de Brazzaville, témoigne de l'exceptionnel niveau de qualité et de la créativité de la langue française dans un pays où le Ministre des hydrocarbures est un poète, lauréat du prix de l'Académie française, et qui compte quelques-uns des écrivains parmi les plus créatifs et novateurs de la littérature francophone de ces vingt dernières années (Tchicaya U Tamsi, Sony Labou Tansi, Sylvain Bemba, Henri Lopes...). »

\section{RÉSUMÉS}

Résumé

Le paradigme de la singerie est tentaculaire. S'il est de notoriété scientifique et médiatique que les grands singes, chimpanzés et gorilles, habitent, à côté des Pygmées, les forêts primaires du bassin du Congo considéré comme leur niche écologique naturelle, il est plus troublant de voir à quel point le primate habite aussi l'imaginaire des explorateurs, voyageurs et romanciers qui le prennent parfois comme personnage emblématique de leur remontée à la source du fleuve. Que leurs récits se penchent sur le berceau de l'humanité, se lancent à la poursuite du chaînon manquant, réfléchissent aux fondements de la violence et de la guerre, ou inventent un langage pour la première victime subalterne de l'homme, la passion de l'origine, aussi indistincte que fascinante, aboutit à confondre l'éthologie et l'ethnologie, à propager une vulgate évolutionniste, à réactiver les schémas de l'anthropologie physique ainsi que les clichés interculturels sur la race et la langue. $\mathrm{Du}$ " patois » au français en passant par le petit-nègre, de la pratique du « symbole » en vigueur à l'école coloniale à la théorie de la « symbiose » francophone, de la bestialité grégaire à la bêtise stéréotypée, le singe apparaît comme le signe crypté d'un éco-système mimétique, ne cessant de monter et descendre sur cet arbre qui cache les racines primitivistes et raciologiques du concept Congo. 
Abstract

The monkey paradigm is a tentacular one. Both science and the media widely report that the major primates such as chimpanzees and gorillas live side-by-side with the Pygmies in the primeval forests of the Congo, considered to be their natural ecological niche. However, the extent to which monkeys also inhabit the imagination of explorers, travellers and writers, and are used by them as symbolic figures in their quest for origins, is far more disturbing. Whether investigating the cradle of civilisation, searching for the missing link, reflecting on the causes of violence and war, or inventing a language for man's first subordinate, the passion for origins-as hazy as it is fascinating-tends to confuse ethnology with ethology, to propagate an evolutionist vulgate, and reactivate principles of physical anthropology as well as inter-cultural clichés about race and language. From provincial dialects to French via petit-nègre pidgin, from the "symbols" of the colonial school to the theory of francophone "symbiosis", and gregarious bestiality to stereotyped stupidity, the monkey is the encrypted sign of a mimesis ecosystem in which it continues to climb up and down the tree that conceals the primitivist and racial roots of the whole "Congo concept".

\section{INDEX}

Keywords : Congo, Brazzaville, Pygmies, Joseph Conrad, Sony Labou Tansi, Henri Stanley, Ethnology/ethology, Evolved/ Evolutionist, Petit-Nègre/pidgin, Primitivism, Explorers' Accounts, Novels, Monkeys, Symbol/symbiosis, Travel

Mots-clés : Congo, Brazzaville, Pygmées, Joseph Conrad, Sony Labou Tansi, Henri Stanley, ethnologie/éthologie, évolué/évolutionniste, francophonie, petit-nègre, primitivisme, récit d'exploration, romans, singes, symbole/symbiose, voyage 